\title{
Adaptações cardíacas fisiológicas induzidas pelo exercício físico em atletas amadores: revisão narrativa
}

\author{
Physiological cardiac adaptations induced by physical exercise in amateur athletes: \\ narrative review
}

Adaptaciones cardíacas fisiológicas inducidas por el ejercicio físico en atletas aficionados: revisón narrativa

Laerte de Paiva Viana Filho ${ }^{1 *}$, João Pedro Costa Apolinário² ${ }^{2}$ Júlia Oliveira do Carmo ${ }^{3}$, Laís Breguez Pascoal $^{4}$, Lucas Pereira Figueiredo ${ }^{5}$, Luísa Lobo Sousa ${ }^{6}$, Milena Oliveira Moreira ${ }^{7}$, Wellington de Jesus Ferreira Junior ${ }^{8}$.

\section{RESUMO}

Objetivo: Compilar evidências científicas recentes sobre as adaptações fisiológicas que ocorrem no coração de atletas amadores. Revisão bibliográfica: A prática de exercícios físicos proporciona um aumento do trabalho cardíaco para suprir a demanda metabólica, o que pode provocar adaptações fisiológicas a depender de vários fatores, como o tipo de exercício realizado, sendo esse predominantemente de força, resistência ou misto. Com a popularização da prática de atividades físicas por pessoas que não dependem do esporte como fonte de renda, os atletas amadores, tornou-se significativo a análise de registros de adaptações cardíacas fisiológicas induzidas pelo exercício que podem ocorrer nesses indivíduos. Com isso, foi possível observar as principais adaptações em praticantes de exercícios de resistência, que incluem as adaptações morfológicas do coração, no sistema de condução do impulso elétrico, além de adaptações bioquímicas e funcionais do sistema cardiovascular. Já as induzidas pelos exercícios de força, incluem predominantemente as adaptações morfológicas e funcionais, enquanto as desenvolvidas pela prática de exercícios mistos incluem alterações funcionais, morfológicas e elétricas. Considerações finais: Percebe-se a necessidade de um maior monitoramento cardiológico em atletas amadores, tendo em vista o risco aumentado de complicações cardiovasculares nesses atletas.

Palavras-chave: Cardiomegalia induzida por exercícios, Exercício físico, Fenômenos fisiológicos cardiovasculares.

\footnotetext{
ABSTRACT

Objective: To compile recent scientific evidence on the physiological adaptations that occur in the hearts of amateur athletes. Bibliographic review: The practice of physical exercises provides an increase in cardiac work to supply the metabolic demand, which can cause physiological adaptations depending on several factors, such as the type of exercise performed, which is predominantly strength, resistance or mixed. With the popularization of the practice of physical activities by people who do not depend on sport as a source of income, amateur athletes, the analysis of records of physiological cardiac adaptations induced by exercise that may occur in these individuals has become significant. With that, it was possible to observe the main adaptations in resistance exercise practitioners, which include the morphological adaptations of the heart, in the electrical impulse conduction system, in addition to biochemical and functional adaptations of the

1Pontifícia Universidade Católica de Minas Gerais (PUC-MG), Betim - MG. *E-mail: laerteviana07@gmail.com ${ }^{2}$ Residente de Clínica Médica do Hospital Madre Teresa (HMT), Belo Horizonte - MG.

${ }^{3}$ Pontifícia Universidade Católica de Campinas (PUC - Campinas), Campinas - SP.

${ }^{4}$ Centro Universitário de Belo Horizonte (UniBh), Belo Horizonte - MG.

5Universidade Federal de Viçosa (UFV), Viçosa - MG.

${ }^{6}$ Centro Universitário Atenas (Uniatenas), Paracatu - MG.

7Universidade Federal de São João Del Rei - Campus Dom Bosco (UFSJ-CDB), São João Del Rei - MG.

8Universidade de Itaúna (UIT), Itaúna - MG.
}

SUBMETIDO EM: 5/2020

ACEITO EM: $6 / 2020$

PUBLICADO EM: $8 / 2020$ 
cardiovascular system. Already those induced by strength exercises, predominantly include morphological and functional adaptations, while those developed by the practice of mixed exercises include functional, morphological and electrical changes. Final considerations: There is a need for greater cardiological monitoring in amateur athletes, in view of the increased risk of cardiovascular complications in these athletes.

Keywords: Cardiomegaly exercise-induced, Exercise, Cardiovascular physiological phenomena.

\section{RESUMEN}

Objetivo: Recopilar evidencia científica reciente sobre las adaptaciones fisiológicas que ocurren en los corazones de los atletas aficionados. Revisión bibliográfica: Práctica de ejercicios físicos proporciona un aumento en el trabajo cardíaco para satisfacer la demanda metabólica, lo que puede causar adaptaciones fisiológicas dependiendo de varios factores, como el tipo de ejercicio realizado, que es predominantemente fuerza, resistencia o mixto. Con la popularización de la práctica de actividades físicas por personas que no dependen del deporte como fuente de ingresos, los atletas aficionados, el análisis de los registros de adaptaciones cardíacas fisiológicas inducidas por el ejercicio que pueden ocurrir en estos individuos se ha vuelto significativo. Con eso, fue posible observar las principales adaptaciones en los practicantes de ejercicios de resistencia, que incluyen las adaptaciones morfológicas del corazón, en el sistema de conducción de impulsos eléctricos, además de las adaptaciones bioquímicas y funcionales del sistema cardiovascular. Los inducidos por los ejercicios de fuerza ya incluyen predominantemente adaptaciones morfológicas y funcionales, mientras que los desarrollados por la práctica de ejercicios mixtos incluyen cambios funcionales, morfológicos y eléctricos. Consideraciones finales: Existe la necesidad de una mayor monitorización cardiológica en atletas aficionados, en vista del mayor riesgo de complicaciones cardiovasculares en estos atletas.

Palabras clave: Cardiomegalia Inducida por el ejercicio, Ejercicio físico, Fenómenos fisiológicos cardiovasculares.

\section{INTRODUÇÃO}

O exercício físico gera estresse fisiológico no organismo ao elevar consideravelmente as demandas energéticas em relação ao repouso. Além disso, provoca modificações morfológicas e funcionais nos praticantes que os capacitam a responder ao estresse provocado pelas atividades físicas que desempenham. As alterações provocadas pelo treinamento físico são muito estudadas e complexas porque envolvem uma interação entre vários sistemas do organismo, como o musculoesquelético, cardiovascular, respiratório, metabólico e o sistema nervoso autônomo (SNA).

Nesse sentido, as atividades físicas podem ocasionar, por exemplo, adaptações específicas na musculatura esquelética, como o aumento da massa muscular; adaptações cardiovasculares morfológicas, como hipertrofia cardíaca, e funcionais, como bradicardias e alterações respiratórias, a exemplo do aumento do consumo de oxigênio (VO2max), o qual pode ser causado pela elevação da taxa metabólica do praticante. O SNA tem a capacidade de modular as interações entre esses sistemas, os quais determinam a capacidade funcional do indivíduo (DE PINHO RA, et al., 2010).

Algumas adaptações cardíacas fisiológicas induzidas pelo exercício físico, como a hipertrofia do ventrículo direito (HVE) podem ter manifestações sobrepostas aos padrões de doenças cardiovasculares como a cardiomiopatia hipertrófica $(\mathrm{CMH})$. A CMH é conceituada como uma HVE sem causa definida e acrescida de um desarranjo de miofibrilas, as quais atuam no mecanismo de contração da musculatura cardíaca, e pode levar à morte súbita, aquela em que não há explicações dentro de uma hora dos sintomas sentidos.

Em um estudo recente realizado sobre a incidência de morte súbita em atletas do National Collegiate Athletic Association foram detectadas 273 mortes em 1964663 atletas durante o período de cinco anos, tendo como causa principal (56\%) alterações cardiovasculares, representando $75 \%$ dos eventos fatais que ocorrem no momento em que o atleta desempenha atividade física. Devido a isso, é de extrema importância diferenciar os dois quadros, identificando alterações cardíacas induzidas pelo exercício físico em atletas que são fisiológicas, e por isso não representam riscos, o que torna relevante o presente estudo (DOS SANTOS FC, et al., 2012). 
A prática de exercício físico age no mecanismo de prevenção e promoção de saúde, podendo desacelerar as alterações fisiológicas do envelhecimento e das doenças crônico-degenerativas, proporcionando adaptações morfológicas e funcionais positivas no músculo cardíaco (GONÇALVES MP e ALCHIERE JC, 2010; LI Y, et al., 2020).

Além disso, é sabido que nos últimos anos houve uma promoção de um estilo de vida saudável e a disseminação de práticas esportivas, como levantamento de peso e crossfit, que são treinamentos potencialmente intensos e que podem levar a condições que colocam a vida do praticante em risco quando não monitoradas corretamente (PIGOLKIN YI, et al., 2019).

Nesse sentido, observa-se que, a curto prazo, o aumento do trabalho cardíaco visa a atender a demanda metabólica de exercícios extenuantes. Já a longo prazo, os exercícios podem provocar a remodelação cardíaca, incluindo crescimento e reprogramação molecular e celular adaptativa fisiológicas, acompanhado por aumento na capacidade de produção de energia, o que contrasta com as adaptações cardíacas patológicas, em que a função contrátil e a produção metabólica de energia diminuem e há um comprometimento funcional cardíaco (GRONEK P, 2020; VEGA RB, et al., 2017).

As alterações cardíacas fisiológicas e estruturais que ocorrem entre os atletas de elite são um fenômeno conhecido como "coração de atleta", e com o crescimento da participação de amadores em competições intensas como maratonas, há uma necessidade maior de avaliar a ocorrência dessas alterações nesses atletas amadores (KALETA AM, et al., 2018). Nesse sentido, cabe explanar que a classificação de atleta amador neste artigo foi pautada na Lei oㅜ 9.615, de 24 de março de 1998, que identifica este como o indivíduo que realiza exercícios físicos sem remuneração, independente do seu desempenho (MIGUEL RGA, 2014).

Considerando essas informações, este trabalho teve como objetivo compilar, através de uma revisão bibliográfica, as evidências científicas mais recentes sobre as principais adaptações fisiológicas que ocorrem no coração de atletas amadores ao serem submetidos a diferentes modalidades de exercícios físicos regularmente.

\section{REVISÃO BIBLIOGRÁFICA}

\section{Adaptações fisiológicas nos exercícios predominantemente de resistência}

Os exercícios físicos de resistência são aqueles que exigem que o atleta mantenha um determinado esforço pelo máximo de tempo possível, envolvendo massas musculares importantes e todo o sistema cardiovascular e respiratório. Nesse sentido, exercícios como corrida e natação dependem de um suprimento maior de oxigênio para os músculos e, por envolverem uma contração rítmica dos músculos esqueléticos, quando executados por um período prolongado (30 a 60 minutos, por exemplo) apresentam melhora no retorno venoso, aumentando dessa forma o volume de sangue no ventrículo esquerdo (VE) antes da contração cardíaca e da ejeção do sangue para a circulação sistêmica, denominado volume diastólico final (VDF) (FERNANDES T, et al., 2015).

Estudos realizados por Sierra APR, et al. (2016) e Montiel G, et al. (2015) observaram também, através de análises ecocardiográficas, que após corridas de maratona, ocorre nos atletas um comprometimento no relaxamento da musculatura cardíaca sem haver, entretanto, uma disfunção diastólica, já que não houveram anormalidades registradas no VDF. O comprometimento observado ocorreu devido à diminuição da velocidade do fluxo de sangue passivo pela valva mitral durante o enchimento do VE - diástole precoce - e pela diminuição da velocidade de fluxo sanguíneo pela valva mitral para o VE na contração do átrio esquerdo (AE) - diástole tardia.

Assim, não há indicações que esse comprometimento represente uma alteração patológica, mas sim uma caracterização do "coração de atleta", o que não gera implicações significativas no funcionamento cardiovascular dos atletas amadores já que o volume de sangue ejetado para a circulação sistêmica, a fração de ejeção do ventrículo esquerdo (FEVE), não se alterou. 
No que tange às alterações bioquímicas do coração, um estudo de Montiel G, et al. (2015) observou que em maratonistas amadores, imediatamente após o exercício, ocorre um aumento na atividade da Troponina $\mathrm{T}$ (cTnT), o que indica uma estimulação aumentada do miocárdio e um estresse temporário desse. Além disso, Legaz-Arrese A, et al. (2015) notou, em remadores amadores, uma elevação de Troponina I cardíaca (cTnl) e de Peptídeo Natriurético Pró-cerebral N-terminal (NT - proBNP).

A troponina é composta por 3 isoformas denominadas T, I e C e, como função, pode-se destacar a participação essencial na regulação da contração cardíaca e muscular. As isoformas cTnl e cTnT são específicas do músculo cardíaco, funcionando como marcadores de lesão miocárdica (DA SILVA SH e MORESCO RN, 2011). Já o NT - proBNP é um marcador resultante da clivagem enzimática do Peptídeo Natriurético Cerebral (BNP) que, segundo Sierra AP, et al. (2015), tem a função de indicar sobrecarga miocárdica funcional, bem como a presença de estresse da parede miocárdica. Em ambos artigos essas elevações foram consideradas adaptações fisiológicas, não indicando necessariamente lesão no miocárdio.

Em relação ao aumento da cTnT imediatamente após exercícios de resistência, observa-se que um tempo após a execução da atividade, a cTnT retorna aos valores pré-exercício (SIERRA AP, et al., 2015). Segundo Da Silva SH e Moresco RN (2011), quando ocorre um dano miocárdico, espera-se que a elevação da troponina seja persistente. Ademais, no estudo de Legaz-Arrese A, et al. (2015), observou-se um aumento da cTnl após 3, 6 e 12 horas do exercício de ultra resistência, havendo retorno quase que a níveis pré-exercício após 24 horas da remada de 30 minutos, ou seja, distinguiu da curva esperada em caso de lesão miocárdica isquêmica.

Por isso, os autores consideraram que o aumento desse biomarcador, na ausência de manifestações clínicas sugestivas de isquemia, seja fisiológico. No que concerne ao aumento do NT - proBNP após exercício físico, ainda há controvérsias na literatura, mas o alto estresse causado pelo exercício de ultra resistência parece ser o responsável pela sua elevação transitória, o que, na ausência de manifestações clínicas sugestivas de doenças cardíacas, é considerado fisiológico (LEGAZ-ARRESE A, et al.,2015). Nesse sentido, cabe mencionar que as troponinas são altamente específicas e sensíveis para o diagnóstico de síndromes coronarianas agudas, porém, é visto que concentrações elevadas desses marcadores após exercícios de longa duração têm sido encontradas sem a presença de sintomas clínicos de infarto agudo do miocárdio.

Os níveis de BNP, que também se encontram aumentados frente ao dano cardíaco, não se elevam em corações fisiologicamente hipertrofiados como o "coração de atleta", mas há evidências que demonstram a existência da elevação transitória desses níveis imediatamente após exercícios prolongados em indivíduos saudáveis.

Desse modo, foi visto que a realização do exercício de resistência promoveu uma elevação significativa dos marcadores BNP e troponina T cardíaca em uma parcela considerável dos praticantes. Isso pode ser justificado pela ocorrência de uma isquemia miocárdica transitória durante o exercício devido a prioridade do fluxo sanguíneo para a musculatura esquelética. A ideia de que a isquemia miocárdica pode causar liberação de troponina deve ser considerada na prática clínica e no exercício. Já o BNP, que é um biomarcador indicativo de sobrecarga miocárdica funcional, reflete o estresse da parede miocárdica (SIERRA AP, et. al., 2015).

Em relação à frequência cardíaca $(\mathrm{FC})$, um estudo de Haaf $\mathrm{TT}$, et al. (2019) realizado com ciclistas observou que ocorre uma diminuição da FC 5 a 8 dias após o aumento da carga de treinamento, em um evento não competitivo de ciclismo amador, com duração de 8 dias. Essas observações sugerem que a duração do treinamento intensificado e o aumento relativo na carga de treinamento interferem para uma FC mais baixa. Em consonância, Kaleta AM, et al. (2018) observou que, em atletas amadores de maratona, a bradicardia sinusal (FC abaixo de 60bpm) foi um achado comum no eletrocardiograma (ECG), principalmente em repouso.

A bradicardia sinusal, variante mais encontrada nos atletas, pode ocorrer devido à elevação do tônus vagal, que é dado pela atividade do sistema nervoso autônomo parassimpático no coração, da diminuição da densidade e/ou sensibilidade dos receptores da adrenal, além da remodelagem do canal iônico do nó sinusal, 
responsável pelo automatismo cardíaco e assim, pelo ritmo de contração cardíaca. Apesar de ser uma característica do "coração de atleta", segundo estudos abordados por Kaleta AM, et al. (2018), a bradicardia sinusal pode desencadear quadros patológicos. Ao relacionar-se com o aumento do tônus vagal, a bradicardia sinusal pode implicar em um quadro de fibrilação atrial, contrações desordenadas dos átrios, além de criar de uma via de reentrada para a transmissão do impulso elétrico no coração.

Em contrapartida, imediatamente após uma corrida de ultradistância $(80 \mathrm{~km})$, atletas amadores apresentaram aumento na FC, segundo Jouffroy R, et al. (2015). Embora a reativação da atividade vagal ocorra rapidamente após o término da atividade física, a atividade do sistema nervoso autônomo simpático permanece aumentada até 25 a 30 minutos após, contribuindo para o aumento observado na FC no pósexercício imediato (PRAZERES TMP, et al., 2017).

Além disso, Jouffroy R, et al. (2015) observou, em seu estudo, um aumento no diâmetro do VE no final da sístole, e diminuição na pressão arterial (PA) sistólica e PA diastólica após a corrida de ultradistância. Nesse sentido, vale ressaltar que a queda pressórica pode ocorrer devido a diminuição no débito cardíaco, na resistência vascular periférica, na atividade neural simpática, além da produção de substâncias vasoativas, como óxido nítrico, prostaglandinas, bradicinina, adenosina e lactato (FROIS RRS, et al., 2012).

Kaleta AM, et al. (2018) identificou ainda outras anomalias no ECG em atletas amadores, antes e após uma maratona. As variantes relacionadas ao treinamento foram mais comuns no repouso e podem ser identificadas por: bloqueio atrioventricular de primeiro grau (BAV $1^{\circ}$ grau), bloqueio de ramo direito incompleto (BRD incompleto), repolarização precoce e hipertrofia ventricular esquerda (HVE). Esses achados configurariam patologias se o critério de análise usado para os exames fosse o geral, mas que são benignos no ECG de pessoas ativas.

A hipertrofia cardíaca é uma resposta adaptativa do coração ao aumento da carga de trabalho e envolve vários fatores mecânicos, hemodinâmicos e hormonais. O principal fator da remodelação do VE é a carga de trabalho cardíaco hemodinâmico. A hipertrofia cardíaca fisiológica pode ser concêntrica ou excêntrica, as quais induzem adaptações distintas no coração, principalmente no VE.

A remodelação do VE na forma de hipertrofia concêntrica fisiológica (adição paralela de novos sarcômeros e crescimento lateral de cardiomiócitos individuais) geralmente leva ao aumento da espessura da parede do VE sem alteração no diâmetro da câmara, sendo considerada induzida pelo treinamento do exercício, assim como a remodelação na forma de hipertrofia excêntrica (adição de sarcômeros em série e crescimento longitudinal de cardiomiócitos), a qual leva ao aumento na dilatação do VE. O remodelamento fisiológico do VE preserva, ou até melhora, a função ventricular, levando a um crescimento coordenado da massa muscular e da angiogênese (FERNANDES T, et al., 2015).

Nesse sentido, a hipertrofia cardíaca fisiológica induzida pelo treinamento físico apresenta efeitos cardioprotetores ao possibilitar uma ampliação da força contrátil do miocárdio e, assim, contrações mais vigorosas do coração. Além disso, essa hipertrofia não está relacionada à insuficiência cardíaca, já que na primeira tem-se uma melhora na eficácia do coração, enquanto na insuficiência cardíaca a capacidade de bombeamento de sangue se encontra comprometida, havendo uma diminuição no retorno venoso e no débito cardíaco (FERNANDES T, et al., 2015).

Dessa forma, as principais adaptações cardíacas fisiológicas induzidas pelo exercício predominantemente de resistência no coração do atleta amador encontradas nos estudos revisados são: alteração da função diastólica, aumento de biomarcadores cardíacos (CK-MB, cTnT, cTnl, NT- proBNP), aumento da FC imediatamente após a execução do exercício, bradicardia sinusal 5 a 8 dias após exercício e em repouso préexercício, redução da PA sistólica e diastólica após o exercício e alterações ao ECG em repouso (BAV 1ำ grau, BRD incompleto, repolarização precoce e HVE). A ocorrência de eventos fatais em atletas praticantes dos exercícios predominantemente de resistência relaciona-se principalmente com a presença de comorbidades cardíacas ocultas ou já existentes que não possuem relação com a prática desses exercícios (BERNARDO M, 2019). 


\section{Adaptações fisiológicas nos exercícios predominantemente de força}

Os exercícios de força, como levantamento de peso, envolvem uma menor massa muscular em comparação aos exercícios de resistência citados anteriormente, mas a contração muscular é levada até a exaustão com um número de repetições geralmente inferior a vinte. A contração isométrica com cargas mais pesadas promove obstrução mecânica do fluxo sanguíneo muscular, levando ao aumento da resistência vascular sistêmica e da pressão arterial (PA sistólica superior a $250 \mathrm{mmHg}$ é comumente encontrada durante esse tipo de exercício) nestes atletas (FERNANDES T, et al., 2015). No entanto, essas variações parecem ser decorrentes dos efeitos agudos pós-exercício, resultantes da ativação do sistema nervoso simpático.

Afinal, quando se refere aos efeitos crônicos, o treinamento de força é eficaz na redução da rigidez arterial periférica, atribuído principalmente a melhorias na função endotelial e na estimulação da produção de substâncias vasoativas. Além disso, a diminuição da PA sistêmica foi descrita como um dos principais efeitos sistêmicos em indivíduos hipertensos praticantes de exercícios de força. Essa diminuição pode ser atribuída, além da redução da resistência vascular periférica, ao aumento na sensibilidade dos barorreceptores e da melhora na perfusão microvascular e/ou na função endotelial (SANTOS J, 2016).

Segundo Ryaboy IV, et al. (2018), houve uma diferença nas dimensões cardíacas de atletas que praticavam exclusivamente treinos de força e atletas que praticavam exercícios de resistência, apesar de todos possuírem um diâmetro interno do VE aumentado. Os atletas de força apresentaram uma média muito maior da espessura relativa do VE $(44 \mathrm{~mm})$ que os de resistência $(39 \mathrm{~mm})$ e o grupo controle $(36 \mathrm{~mm})$. Da mesma forma, atletas de força tiveram uma espessura septal igual a $11,8 \mathrm{~mm}$, enquanto os de resistência apresentaram espessura de $10,5 \mathrm{~mm}$ e o grupo controle $8,8 \mathrm{~mm}$.

Segundo Bernardo M (2019), o aumento da espessura da parede do VE superior ou igual a $13 \mathrm{~mm}$ sem doenças cardíacas ou sistêmicas associado a um aumento na espessura do septo interventricular podem indicar cardiomiopatia hipertrófica $(\mathrm{CMH})$. No entanto, os achados apontados no estudo de Ryaboy IV, et al. (2018) vão de encontro a tais valores, demonstrando crescimentos benignos e comuns no atleta que, se não acompanhados de disfunções, não torna necessária a interrupção da prática esportiva, justamente por não impactar negativamente na saúde do praticante que sofre essas alterações.

Assim, as principais adaptações cardíacas fisiológicas induzidas pelo exercício predominantemente de força no coração do atleta amador encontradas nos estudos revisados são: aumento da resistência vascular sistêmica e da PA sistólica, principalmente nos momentos pós-exercício, além do aumento da espessura do VE e do septo interventricular.

\section{Adaptações fisiológicas nos exercícios combinados de resistência e força}

O treinamento físico de resistência combinado ao exercício de força induz inúmeras alterações na morfologia e função cardíaca, representando uma resposta adaptativa fisiológica em consequência ao aumento recorrente na carga hemodinâmica. Nesse sentido, um estudo realizado por Rojek A, et al. (2015) observou que no período pós-treinamento de atividades de resistência como: corrida, ciclismo, natação e atividades de força como: levantamento de peso, flexões e treinamento de salto, os atletas amadores apresentam uma FC mais baixa em relação ao nível basal.

Além disso, o mesmo estudo evidenciou algumas alterações ecocardiográficas encontradas no póstreinamento, como: aumento significativo no diâmetro e VDF do VE, aumento na dimensão do átrio esquerdo e ausência de alterações no diâmetro do ventrículo direito (VD). A melhora da função diastólica do VE foi justificada por meio do relaxamento e complacência do miocárdio com recaptação aumentada de cálcio pelo retículo sarcoplasmático, associado a diminuição de tecido conjuntivo e da apoptose dos cardiomiócitos induzido pelo treinamento regular. Já a função sistólica do VE demonstrou ser um pouco maior somente durante o programa de treinamento.

Em contrapartida, observou-se uma diminuição significativa no desempenho sistólico do VD, indicado pela menor deformação miocárdica durante o treinamento. $O$ estudo com atividade física regular realizado por período de 12 meses modificou tanto o desempenho do VE quanto do VD em repouso, em indivíduos 
previamente não treinados. As diferentes respostas do ápice e da base do VE, com redução da contratilidade do primeiro e aumento da rotação do último, desempenham um papel fundamental na remodelação do coração de atleta e representam um mecanismo protetor que reduz o estresse miocárdico (ROJEK A, et al., 2015).

O estudo observacional realizado por Jongman JK, et al. (2015) avaliou a prevalência de anormalidades ao ECG médio com sinal anormal e nas dimensões do VD em atletas que competem em disciplinas esportivas com componentes combinados de força e resistência (endurance).

O eletrocardiograma com média de sinais (SAECG) é uma técnica eletrocardiográfica especial, na qual vários sinais elétricos do coração são calculados para remover interferências e revelar pequenas variações no complexo QRS (representação eletrocardiográfica da despolarização ventricular), chamados de "potenciais tardios".

Nos resultados, a maioria dos atletas revelaram potenciais tardios no SAECG, sendo o prolongamento do fQRS (fragmentação do QRS em pelo menos duas derivações contíguas) a alteração mais expressiva, o que leva, de acordo com critérios da Força-Tarefa de 2010, ao diagnóstico de cardiomiopatia arritmogênica do VD (ARVC). Foi observado que a extensão do prolongamento do fQRS está positivamente correlacionada com as dimensões do VD.

Portanto, os achados do SAECG, ou seja, o prolongamento da duração do fQRS, devem ser interpretados com cautela, pois parece provável que o prolongamento do fQRS seja um fenômeno fisiológico benigno em atletas.

Diante disso, as principais adaptações cardíacas fisiológicas induzidas pelo exercício de resistência e força no coração do atleta amador encontradas nos estudos revisados são: diminuição da FC em repouso após o exercício, aumento do diâmetro, índice de massa e VDF do VE, aumento da função sistólica e melhora na função diastólica do VE, elevação da contratilidade apical e aumento da rotação da base do VE.

Houve também um registro de adaptações relacionadas ao aumento do $\mathrm{AE}$, aumento da espessura do septo interventricular e queda no desempenho sistólico do VD. Além disso, observou-se, na análise de SAECG, um prolongamento do fQRS relacionado à dimensão do VD.

\section{CONSIDERAÇÕES FINAIS}

Assim, com base nos resultados observados, o presente estudo contribui para alertar sobre a importância de um acompanhamento médico frequente dos atletas amadores. Além disso, demonstra a necessidade da identificação e diferenciação, pelos profissionais de saúde, de adaptações fisiológicas características do "coração de atleta" das alterações patológicas, como o aumento da espessura da parede do VE e CMH, apesar das barreiras entre o fisiológico e o patológico não serem muito bem definidas. Tais identificações podem ser feitas através de exames como ECG, ecocardiograma e ressonância magnética e incluem: adaptações cardíacas morfológicas, como as hipertrofias; funcionais, como as alterações na FC; e bioquímicas, como o aparecimento, ainda que transitório, de biomarcadores de lesão cardíaca, a exemplo da troponina T. A ausência de acompanhamento médico e realização de exames antes e durante a prática de exercício por amadores torna os casos de morte súbita muito mais frequentes que em profissionais, os quais possuem uma supervisão médica contínua, reduzindo os riscos de complicações fatais pela prática de exercícios físicos.

\section{REFERÊNCIAS}

1. BERNARDO M. Alterações eletrocardiográficas no atleta e preditores da morte súbita cardíaca. Dissertação (Mestrado Integrado em Medicina) - Instituto de Ciências Biomédicas Abel Salazar. Universidade do Porto, Porto, 2019. 
2. DA SILVA SH, MORESCO RN. Biomarcadores cardíacos na avaliação da síndrome coronariana aguda. Scientia medica, $2011 ; 21(3): 132-142$.

3. DE PINHO RA, et al. Doença arterial coronariana, exercício físico e estresse oxidativo. Arq Bras Cardiol, 2010; 94.4 : 549-55.

4. DOS SANTOS FC, et al. Morte súbita cardíaca em atletas. Revista da Faculdade de Ciências Médicas de Sorocaba, 2012; 14(4): 131-134.

5. FERNANDES T, et al. Aerobic exercise training promotes physiological cardiac remodeling involving a set of microRNAs. American Journal of Physiology-Heart and Circulatory Physiology, 2015; 309(4): 543-552.

6. FROIS RRS, et al. Hipotensão pós-exercício: influência da modalidade e intensidade do exercício sobre as respostas hipotensoras agudas. Brasília Med, 2012;49(2):98-103.

7. GONÇALVES MP, ALCHIERI JC. Motivação à prática de atividades físicas: um estudo com praticantes não-atletas. Psico-USf, 2010; 15(1): 125-134.

8. GRONEK P, et al. A Review of Exercise as Medicine in Cardiovascular Disease: Pathology and Mechanism. Aging and disease, 2020; 11(2): 327-340.

9. HAAF TT, et al. Submaximal heart rate seems inadequate to prescribe and monitor intensified training. European Journal of Sport Science, 2019; 19(8): 1082-1091.

10. JONGMAN JK, et al. Relationship between echocardiographic right-ventricular dimensions and signal-averaged electrocardiogram abnormalities in endurance athletes. Ep Europace, 2015; 17(9): 1441-1448.

11. JOUFFROY R, et al. Changes of Cardiac Function During Ultradistance Trail Running. American Journal of Cardiology, 2015; 116(8): 1284-1289.

12. JUNIOR ND, SANTOS GG. Efeitos induzidos pelo treinamento de força: Revisão sobre as alterações fisiológicas em indivíduos hipertensos. Semina: Ciências Biológicas e da Saúde, 2016; 37(2): 107-114.

13. KALETA AM, et al. Electrocardiographic abnormalities in amateur male marathon runners. Advances in Clinical and Experimental Medicine, 2018; 27(8): 1091-1098.

14. LEGAZ-ARRESE A, et al. Individual variability in cardiac biomarker release after 30 min of high-intensity rowing in elite and amateur athletes. Applied Physiology, Nutrition, and Metabolism, 2015; 40(9): 951-958.

15. LI Y, et al. Healthy lifestyle and life expectancy free of cancer, cardiovascular disease, and type 2 diabetes: prospective cohort study. BMJ, 2020; 368.

16. MIGUEL RGA. Atleta: definição, classificação e deveres. Revista eletrônica [do] Tribunal Regional do Trabalho da 9a Região, Curitiba, PR, 2014; 3(29): 51-61.

17. MONTIEL G, et al. Echocardiographic and biochemical analysis of cardiac function and injury among female amateur runners post-marathon. Wiener klinische Wochenschrift, 2016; 128(5-6): 193-197.

18. PIGOLGKIN YI, et al. Sudden death of young people with various types of physical activity. Forensic Medicine, 2019; 62(1): 50-55.

19. PRAZERES TMP, et al. Cardiovascular responses during resistance exercise after an aerobic session. Brazilian Journal of Physical Therapy, 2017; 21(5): 329-335.

20. ROJEK A, et al. Biventricular response of the heart to endurance exercise training in previously untrained subjects. Echocardiography. Mount Kisco N.Y, 2015; 32:779-86.

21. RYABOY IV, et al. Aviator's Heart: A Case of Athlete's Heart in an Active Duty Male Naval Aviator. Military medicine, 2018; 183(11-12): e783-e786.

22. SIERRA AP, et al. Alteração de biomarcadores de lesão miocárdica em atletas após a Maratona Internacional de São Paulo. Revista Brasileira de Medicina do Esporte, 2015; 21(3): 182-186.

23. SIERRA APR, et al. Reduction in post-marathon peak oxygen consumption: sign of cardiac fatigue in amateur runners? Arquivos brasileiros de cardiologia, 2016; 106(2): 92-96.

24. VEGA RB, et al. Mecanismos moleculares subjacentes à adaptação cardíaca ao exercício. Cell metabolism, 2017; 25(5): 1012-1026. 